\title{
Entornos Tecnológicos y su Influencia en los Espacios de Trabajo Matemático
}

\section{Technological environments and its influence in Mathematical Working Spaces}

\author{
Antonio Codina Sánchez ${ }^{*}$ \\ Isabel $\mathrm{M}^{\mathrm{a}}$ Romero Albaladejo ${ }^{* *}$
}

\begin{abstract}
Resumen
Este trabajo analiza la influencia de contextos ricos en tecnología digital sobre los Espacios de Trabajo Matemático (ETM). En particular, pretende poner de manifiesto cómo el uso de la tecnología propicia, por una parte, la modificación de roles en los agentes implicados en el proceso de enseñanza-aprendizaje y, por otra, la aparición de ETM compartidos. Utilizando el enfoque de la metodología observacional y el trabajo colaborativo en resolución de problemas como estrategia didáctica con estudiantes para maestro, se ilustrará: (a) cómo influye la interactividad con una i-actividad y el uso de herramientas de comunicación asincrónicas en la construcción de ETM compartidos, y (b) cómo la i-actividad y los estudiantes asumen parcialmente el rol del docente en la gestión de ETM personales y compartidos, ayudados éstos por la tecnología digital.
\end{abstract}

Palabras Clave: Espacios de Trabajo Matemático. Entornos Tecnológicos. Interactividad. Trabajo Colaborativo. Resolución de Problemas.

\begin{abstract}
This work analyzes the influence of technologically rich environments on Mathematical Working Spaces (MWS). In particular, it aims to show how the use of technology fosters, on one hand, changes in the role adopted by agents of the teaching-learning processes, and, on the other hand, the arising of shared MWS. Using observational methodology and collaborative work on problem solving as a didactical strategy with student teachers, we shall illustrate: (a) how the interactivity with an i-activity and the use of asynchronous communication tools influences the construction of shared MWS; and (b) how the i-activity and the students partially take the instructor's role in the management of Personal and shared MWS, aided by digital technology.
\end{abstract}

Keywords: Mathematical Working Spaces. Technological Environments. Interactivity. Collaborative Work. Problem Solving.

\footnotetext{
* Doctor en Didáctica de la Matemáticas, Universidad de Granada (UGR). Universidad de Almería (UAL), Almería, España. Facultad de Educación, Enfermería y Fisioterapia, La Cañada de San Urbano s/n, C.P. 04120, Almería.acodina@ual.es

** Doctora en Didáctica de la Matemática, Universidad de Granada (UGR). Universidad de Almería (UAL), Almería, España. Facultad de Educación, Enfermería y Fisioterapia, La Cañada de San Urbano s/n, C.P. 04120, Almería.imromero@ual.es
} 


\section{Introducción}

La propuesta de los Espacios de Trabajo Matemático (ETM) tiene su antecedente en la teoría de los Espacios de Trabajo Geométrico (ETG) (HOUDEMENT; KUZNIAK, 2006; KUZNIAK, 2005, 2011). Los ETG se articulan en dos niveles o planos, uno de naturaleza epistemológica y otro de naturaleza cognitiva. En el plano cognitivo consideran tres procesos: la visualización-percepción, la construcción y la prueba; mientras que en el plano epistemológico, se consideran tres componentes, el espacio real y local, los artefactos y el marco de referencia. El trabajo matemático permite la articulación entre estos dos niveles gracias a diferentes génesis: la figural o semiótica, la instrumental y la discursiva. Todo ello se conjuga en relaciones dinámicas inter e intra planos (Figura 1).

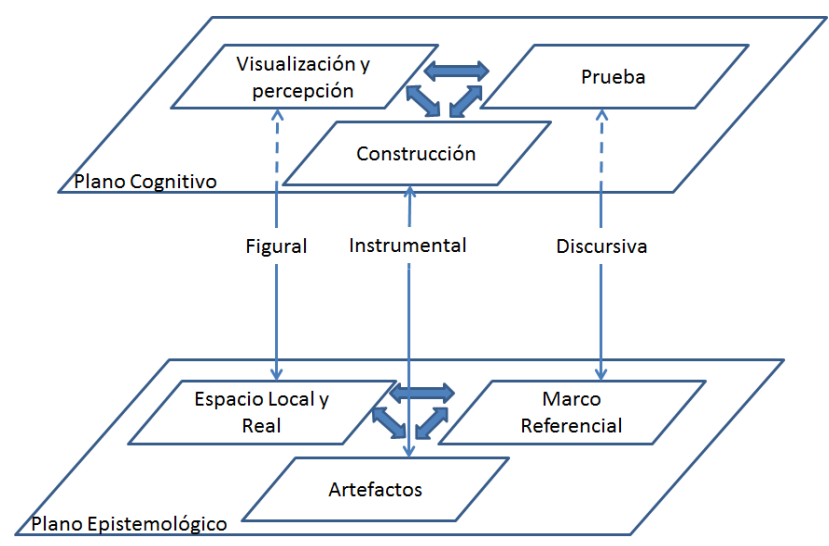

Figura 1 - Proceso dinámico del ETG

Por otro lado, el trabajo matemático en un marco escolar puede describirse gracias a tres niveles de ETM: La matemática considerada por la institución, descrita en el ETM de referencia y desarrollada por el profesor hasta alcanzar un ETM idóneo que permita un establecimiento efectivo en clase, donde cada alumno trabaja en su ETM personal (KUZNIAK; RICHARD, 2014). Estos autores afirman que el diseñador del ETM idóneo desempeña un rol parecido al arquitecto que diseña un ETM para usuarios potenciales. Cuando una tarea se propone a un alumno, el tratamiento matemático depende del ETM personal del alumno. Es por ello que el ETM idóneo no es fijo, sino que debe adaptarse continuamente a los ETM personales de los estudiantes. A este respecto, Montoya (2011) y Barra et al. (2012), sostienen que en la construcción del ETM personal de un estudiante intervienen de forma implícita el docente, los compañeros y la institución. Ello conlleva una relación-tensión entre los ETM personales de los estudiantes, el de referencia y el idóneo. Una 
cuestión aún por explorar es la dinámica de dichas tensiones. En este trabajo contribuimos a ilustrar esta dinámica en ambientes tecnológicos.

En trabajos precedentes (MITHALAL, 2014; GARCÍA; ROMERO; GÓMEZCHACÓN, 2015), se pone de manifiesto la importancia de la interacción social en entornos tecnológicos. En este artículo, proponemos que determinados usos de la tecnología en ambientes de clase colaborativos y de e-learning, hace posible que las herramientas tecnológicas: (a) trasciendan el papel de meros artefactos y asuman parcialmente el papel del profesor en el establecimiento del ETM idóneo, no solo a priori, sino también de forma local y dinámica (KUZNIAK; RICHARD, 2014); (b) generen la posibilidad de construir espacios de trabajo compartidos en los que, de nuevo, el papel de establecer el ETM idóneo no recae exclusivamente en el docente, sino que se distribuye entre los integrantes de la clase. Con este trabajo pretendemos realizar un aporte a la cuestión planteada por Kuzniak y Richard (2014) en relación a cómo la utilización de entornos tecnológicos influye en la construcción epistemológica del alumno, que guía su trabajo matemático.

Para abordar nuestro propósito, nos situamos en el contexto de una asignatura optativa para el grado de Maestro, impartida bajo una modalidad de enseñanza e-Learning. Las directrices generales de la materia ponen especial énfasis en el papel de los estudiantes, subrayando la necesidad de que sean ellos mismos, a través del uso de la tecnología y de la interacción con los compañeros/as y con el formador, quienes ejerzan de constructores de sus propios conocimientos (ETM personales). Además, se pretende que contribuyan a la construcción de un conocimiento común, recogido a través de los medios que proporciona el entorno tecnológico, lo que nos lleva a postular un ETM compartido. La materia se articula en torno a distintos tipos de actividades. De ellas, en este artículo nos referiremos a la resolución de problemas con apoyo de tecnología (Excel, GeoGebra, i-actividades, etc.) y la participación en el Foro de la asignatura.

\section{Marco teórico}

Partiendo del modelo propuesto por Houdement y Kuzniak (2006) para el caso del ETG, diversos autores están identificando los paradigmas asociados para otras áreas de matemáticas. Por ejemplo, Estrella, Kuzniak, Montoya y Vivier (2015) en relación al Espacio de Trabajo de Análisis Matemático, o Mena y Morales (2011) para el Espacio de Trabajo Algebraico muestran como dichos espacios no son una generalización del ETG, detectando 
una naturaleza propia de dichas áreas de contenido. De manera natural surge entonces la noción de ETM, no como una generalización de los ETG, sino como una extensión metodológica (KUZNIAK, 2011; KUZNIAK; RICHARD, 2014). Esta consideración permite a los investigadores tratar los ETM con flexibilidad para cada dominio matemático y las particularidades de los paradigmas asociados. Ello es válido en el campo de la resolución de problemas matemáticos, dónde se interrelacionan distintos ETM.

\subsection{ETM y fases del trabajo matemático en la resolución de una tarea}

En el afán por construir una teoría de los ETM que generalice los saberes adquiridos en la investigación sobre los ETG, los planos verticales de descubrimiento, validación y modelización, introducidos por Coutat y Richard (2011) para el caso particular de la geometría en ambientes tecnológicos, son conectados por Kuzniak y Richard (2014) con las diferentes etapas del trabajo matemático durante la resolución de una tarea o un problema: descubrimiento-exploración, razonamiento-justificación y presentación-comunicación (Figura 2). El trabajo en el plano correspondiente a la primera etapa, con el objetivo de descubrir la solución del problema, privilegia la identificación y exploración a través de las génesis semiótica e instrumental. El trabajo en el plano correspondiente a la segunda etapa, se apoya en el razonamiento matemático, en la justificación de los descubrimientos a través de las génesis instrumental y discursiva. El trabajo matemático en el plano correspondiente a la tercera etapa, presentación-comunicación, está orientado a la presentación matemática de la solución y se apoya en las génesis semiótica y discursiva. 


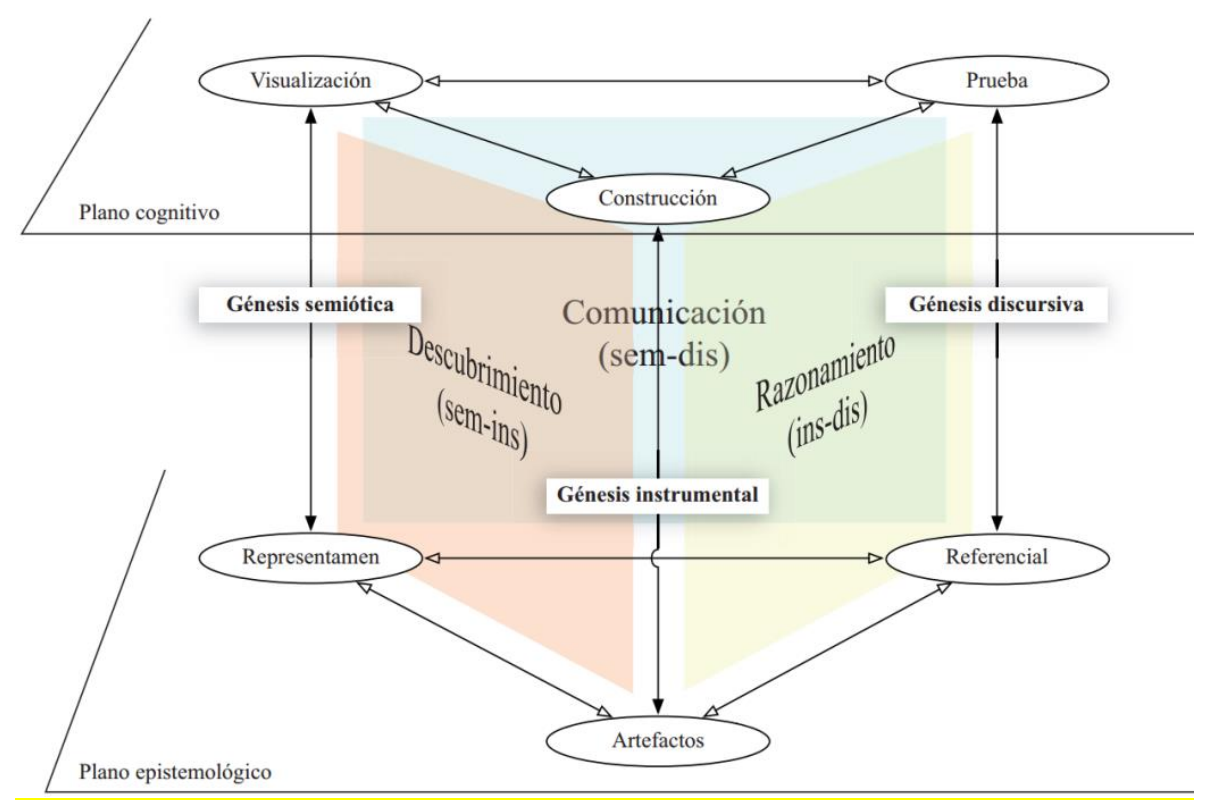

Figura 2 - Planos verticales. Fuente: (KUZNIAK; RICHARD, 2014, p. 13)

\subsection{ETM y tecnología digital}

Barrera (2013) interrelaciona la teoría de la mediación semiótica con la teoría de los ETM al considerar los signos de los artefactos (tecnológicos o no) como mediadores en el aprendizaje compartido. La autora señala que la mediación semiótica tiene especial influencia en la génesis figural. El significado de los objetos matemáticos surge como resultado de la presencia de interacciones semióticas complejas (incluidas el tratamiento con los signos producidos por los artefactos) que son puestas en juego en un plano intermedio entre el epistemológico y el cognitivo (Figura 3). Paralelamente, Gómez-Chacón y Kuzniak (2015) añaden que la introducción de ambientes tecnológicos en las actividades geométricas, junto a la mediación semiótica, promueven, además de la génesis figural, la génesis instrumental y, en menor medida, la discursiva, en ese mismo plano intermedio. 


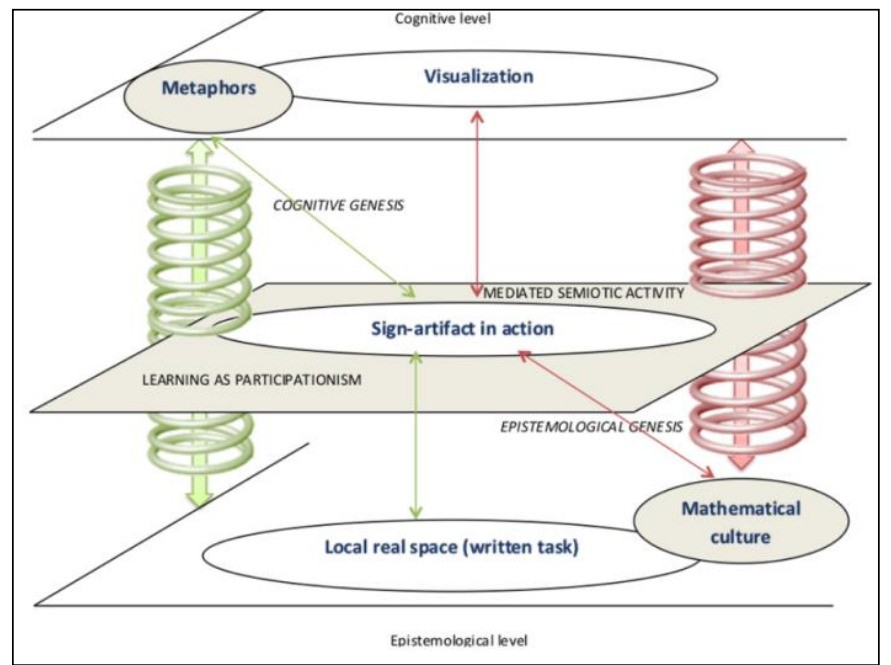

Figura 3 - Aspectos dinámicos en un contexto de mediación semiótica. Fuente: (BARRERA, 2013, p. 5)

Ello es consistente con las ideas expuestas por Wertsch (1991) en relación a que toda actividad cognitiva está mediada por instrumentos. Entonces, la tecnología digital debe considerarse como un instrumento de mediación en la construcción y estructuración de los procesos cognitivos. Gadanidis y Geiger (2010) dan un paso más allá al considerar las tecnologías digitales como agentes cognitivos y no simples herramientas que se utilizan intencionalmente. Desde esta perspectiva, parte del conocimiento construido por el estudiante se deriva de las respuestas que el instrumento tecnológico proporciona a sus acciones intencionales. No obstante, el estudiante reacciona también a la producción de signos por parte de la tecnología digital derivadas de acciones no intencionales. Esto supone que parte del conocimiento del estudiante durante la construcción de su ETM personal pueda considerarse como gestionado por la tecnología. El trabajo de Tessier-Baillargeon et al. (2014) ahonda en esta idea, mostrando como el tutorial inteligente geogebraTUTOR es, más que un medio de ayuda, un espacio de trabajo geométrico idóneo que mediante sus interacciones con los estudiantes, dirige sus conocimientos y es fuente de construcción de nuevos saberes.

Considerando la tecnología como una componente esencial del ETM, las interacciones entre los instrumentos tecnológicos y los signos tienen un papel fundamental, ya que afectan a la construcción epistemológica en el estudiante. Como herramienta cognitiva, "el papel de tales instrumentos va más allá de servir de prótesis para la acción. La presencia de tales instrumentos puede re-organizar todo el funcionamiento cognitivo" (ROJANO; MORENO, 1999, p. 331). En este sentido, la tecnología digital no solo facilita los procesos mentales, sino que es a través de la interactividad que los transforma, entendido el concepto de interactividad como la cualidad de aquellas tecnologías (software o instrumentos tecnológicos) que permiten la interacción, a modo de diálogo, entre usuarios y los artefactos. 
En nuestro caso, consideramos un tipo particular de tecnología digital, las denominadas i-actividades, definidas como actividades interactivas en formato web cuyo objetivo es facilitar y guiar el progreso en la resolución de la actividad y el consiguiente aprendizaje a través de la interacción del estudiante con el ordenador (CODINA; CAÑADAS; CASTRO, 2015). El anterior planteamiento implica que la naturaleza de los significados construidos en los ETM personales no solo es mediada por el profesor, sino también por la influencia de la interactividad con la i-actividad.

\subsection{ETM e interacción social en ambientes e-Learning}

Partimos de la base que los contextos e-Learning no pueden replicar modelos presenciales, sino que es necesario replantear las propuestas didácticas hacia modelos centrados en el autoaprendizaje y donde la interacción social cobra mayor sentido (CODINA, 2009). La idea central recae en la importancia de los procesos de interacción, constituyéndose éstos en elementos clave para la construcción de conocimiento y el aprendizaje (JOCHEMS; MARTENS; STRIJBOS, 2004). Dichas interacciones son consideradas colaborativas cuando para la realización de una actividad los estudiantes aceptan corresponsabilidades, tanto de sus acciones individuales como grupales. En estos ambientes de aprendizaje, la división de tareas es horizontal con un fuerte compromiso para alcanzar el objetivo común de la actividad (DILLENBOURG, 1999).

Admitiendo que "el grado de interacción entre las parejas [de estudiantes] no está definido por la frecuencia de las interacciones, sino por el grado de influencia de esas interacciones en los procesos cognitivos de las parejas" (WALDEGG, 2002, p. 8), los ETM personales tienen que ser analizados a posteriori y observar qué interacciones realmente ocurrieron durante la construcción de éstos (DILLEMBOURG, 1999). Por tanto, hay que observar cómo las ideas se mejoran, refinan o estabilizan a lo largo de la interacción social puesta en juego (MITHALAL, 2014).

Así pues, el horizonte didáctico del formador es la promoción de dichas interacciones sociales, lo que puede lograrse mediante una estrategia metodológica basada en la retroalimentación entre los estudiantes, así como entre éstos y el formador. Parece lógico asumir que estas interacciones permitirán establecer nexos de unión entre los ETM personales e idóneo, al igual que fomentarán las distintas génesis entre los planos cognitivos y epistemológicos de dichos ETM. 
En ambientes presenciales, Barrera (2013) y Gómez-Chacón y Kuzniak (2015) destacan la importancia de la mediación del profesor para que la diversidad de ETM personales de los estudiantes, en un contexto de aula, pueda ir dirigiéndose hacia ETM idóneo. Por su parte, Figueras, Flores y Pluvinage (2014) identifican las dificultades que el docente encuentra en dichos procesos de mediación. Ahora bien, tal como señalan Miskulin et al. (2011), en contextos de e-learning, los estudiantes tienen la oportunidad de construir y profundar en su conocimiento matemático, compartiendo las ideas con el grupo-clase y recibiendo y formulando realimentación y crítica de los compañeros y del profesor. Es decir, en ambientes e-learning los estudiantes asumen un papel más activo en la construcción de sus propios ETM personales y, especialmente, en la contribución a los ETM de los compañeros, asumiendo parcialmente el rol del docente en la gestión del ETM idóneo. Ello implica transformaciones en el papel del docente, que pasa de ser el único mediador entre los ETM puestos en juego (ETM de referencia, ETM idóneo del docente y los ETM personales de los estudiantes), a ejercer un papel de orientador y gestor de las dinámicas de la interacción social entre los estudiantes durante la construcción de sus ETM personales, centrando sus esfuerzos en la creación de un espacio de trabajo en el que los alumnos encuentran y generan condiciones para construir y compartir significados (ZAMBRANO; MEDINA; GARCÍA, 2010).

\section{Objetivos}

Este trabajo pretende poner de manifiesto cómo el uso de la tecnología propicia: (a) la modificación de roles en los agentes implicados en el proceso de enseñanza-aprendizaje, es decir, formador, estudiante(s) y tecnología (digital), y (b) la creación de ETM compartidos. Se ilustrará:

- El papel de una i-actividad como agente cognitivo, asumiendo en parte el papel del formador, y su influencia en la construcción de ETM personales y compartidos.

- La modificación del papel de estudiantes y formador en la construcción de los distintos niveles de ETM (idóneo, personales y compartido), a través de una herramienta de comunicación asincrónica (Foro), y la influencia de las interacciones sociales en este proceso. 


\section{Método}

Este estudio se contextualiza en la asignatura optativa del grado de Maestro "Nuevas Tecnologías en la Enseñanza y Aprendizaje de las Matemáticas" impartida bajo un modelo semipresencial y cursada por 68 estudiantes de diversas titulaciones. La metodología didáctica se apoya en el uso de tecnologías para la resolución de problemas de forma colaborativa. Para este estudio, nos centramos en dos focos de interés. Por una parte, el trabajo en parejas en torno a una i-actividad y, por otra, el uso del Foro como herramienta de comunicación asincrónica para la resolución colaborativa de diversos problemas por parte de los miembros de la clase. A continuación, para cada uno de los focos, describiremos la metodología empleada, tanto a nivel didáctico como de investigación.

\section{1 i-actividad}

Los estudiantes de la asignatura trabajaron por parejas en la resolución de un problema de optimización en formato de i-actividad, enunciada en la Figura 4:

Los puntos $A$ y $F$ son los vértices de una habitación con las siguientes dimensiones: $A B=4 m, B C=3 m$ y $B E=2 m$. Una hormiga está sobre el vértice $A$ de la habitación. ¿Qué camino debe seguir la hormiga, desplazándose por las paredes, para recorrer la menor distancia si desea llegar a punto F?

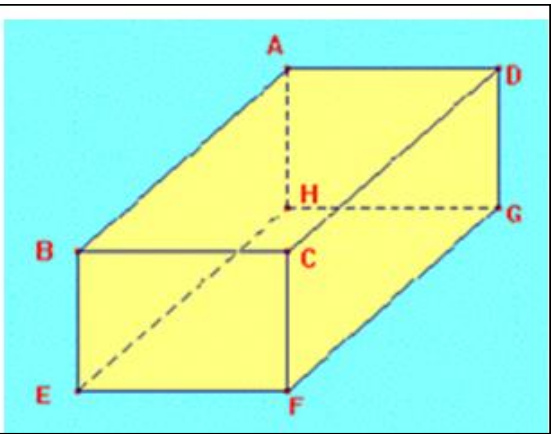

Figura 4 - Enunciado problema de la Hormiga

La i-actividad consta de 6 páginas interconectadas que siguen un diseño Lineal (hay una secuencia intencional de presentación del contenido) e incorporan diversos elementos interactivos (SIMS, 1997): de Objeto (preguntas que se activan cuando se sitúa el cursor sobre ellas); de Actualización (applets para fomentar el diálogo entre estudiante y el contenido generado por la i-actividad); y de Hipervínculo (enlaces a otras páginas de la i-actividad). Además, el flujo de la interacción es continuo, es decir, el estudiante percibe simultáneamente la relación entre su acción y la reacción de la i-actividad (SEDIG; SUMMER, 2006).

En cuanto a los applets, el primero (applet 1) está diseñado para superar el obstáculo de que solo se tengan en cuenta rutas que pasan por los vértices del paralelepípedo; el segundo applet (applet 2) está diseñado para ofrecer una estrategia heurística (levantar el 
"techo" del paralelepípedo); y el tercero (applet 3) está diseñado para superar el obstáculo de considerar sólo rutas por la cara del paralelepípedo que se ve de frente (BCEF) en la representación proporcionada (Figura 5). Esta construcción de la i-actividad confiere al entorno un estatus de ETM idóneo a priori para la resolución del problema de optimización.

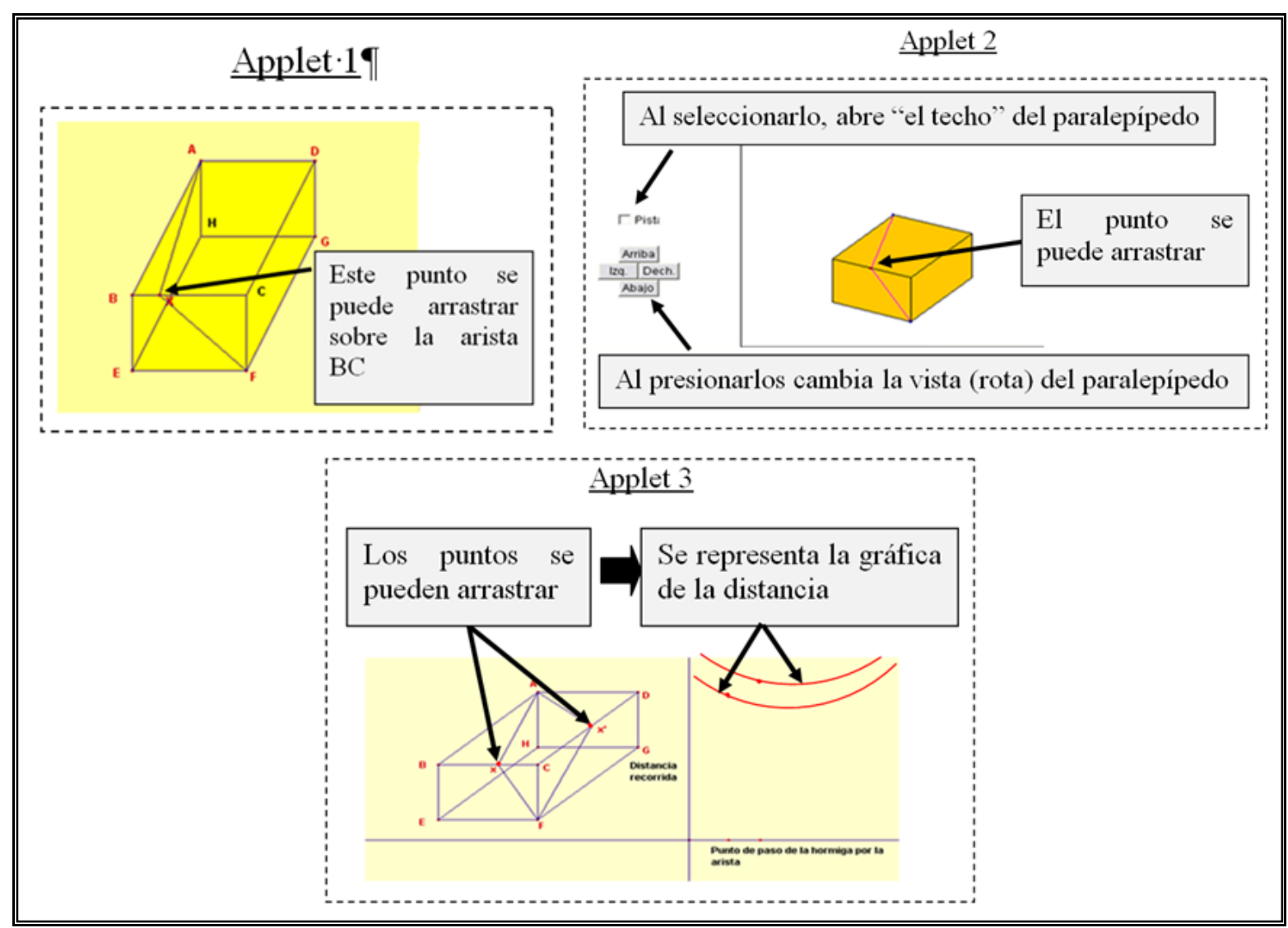

Figura 5 - Applets de la i-actividad

Siguiendo la metodología observacional (ANGUERA, 2010), se seleccionó una muestra de cuatro parejas de entre los estudiantes del curso, que fueron videograbados resolviendo la i-actividad. Dichas grabaciones son transformadas a dos nuevos soportes que facilitan la observación, registro y toma de datos (Figura 6).

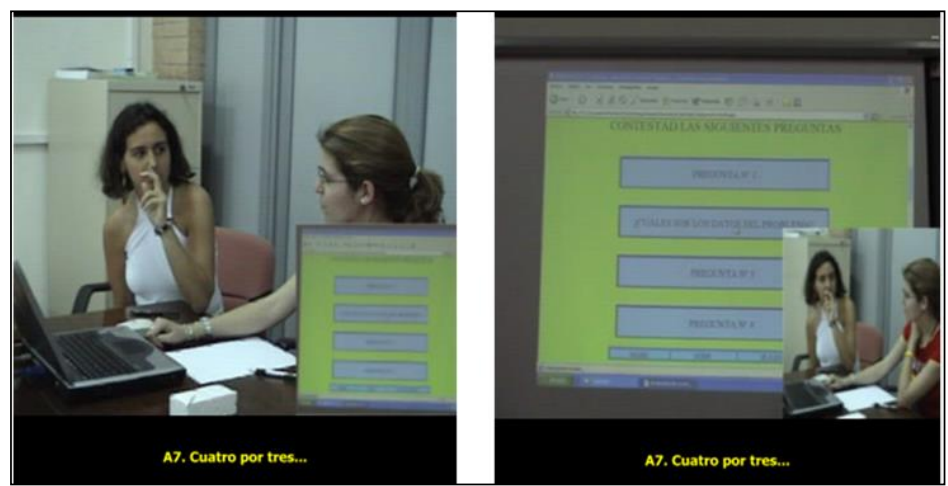

Figura 6 - Fotogramas de los videos para la toma de datos 


\subsection{La resolución de problemas a través del Foro}

El segundo tipo de actividad que es objeto de estudio en este trabajo consiste en la resolución de problemas matemáticas con distintos software. Este trabajo se realiza de manera virtual y se organiza en tres momentos de ejecución que fomenta la interacción social entre los estudiantes:

(a) Momento 1. Los estudiantes envían, leen, analizan y comentan los mensajes del Foro mientras realizan o modifican sus propios trabajos, en un proceso dinámico de intercambio de información. Los estudiantes reciben realimentación valorando y siendo valoradas las versiones preliminares de los trabajos. En situaciones puntuales, el formador interviene para reconducir o precisar informaciones.

(b) Momento 2. Aquellos estudiantes que consideran que su trabajo alcanza los objetivos establecidos, envían su versión al Foro, realizan el test asociado y citan expresamente qué mensajes/trabajos les han sido útiles.

(c) Momento 3. Evaluación por parte del formador. El estudiante envía su trabajo al formador, quien la valora teniendo también en cuenta las citas realizadas a compañeros/as y las que otros compañeros/as hacen del trabajo. El formador envía al estudiante su valoración y realiza comentarios generales. Los estudiantes, tomando como base esta valoración, emiten sugerencias de mejora al Foro.

El análisis de este segundo foco se basa en los procesos acontecidos en el desarrollo de la materia, tal y como tuvieron lugar, desde una perspectiva de la investigación natural (MOSCHOKOVICH; BRENNER, 2000). Para ello, todos y cada uno de los mensajes emitidos en el Foro se archivaron en una base de datos creada ad hoc. Dichos mensajes fueron agrupados atendiendo al contenido semántico y a la influencia sobre el modo de proceder de los compañeros/as en el proceso de resolución. Para la agrupación en función de la componente semántica, se tuvo en cuenta la interacción social fomentada por el mensaje en el Foro. Dicha interacción social trascurre durante los tres momentos de ejecución anteriormente mencionados.

Se analizaron episodios de clase como unidades con sentido matemático completo y se hicieron dos tipos de análisis: el primero centrado en la cesión del rol del profesor como generador/gestor del ETM idóneo a otros agentes (i-actividad o estudiantes), y el segundo centrado en detectar indicios de la posible existencia de un ETM compartido, así como de movimientos entre los distintos niveles de ETM (idóneo, personal y compartido). 


\section{Análisis y discusión}

Analizamos en este apartado, para cada uno de los focos señalados en el apartado anterior (trabajo en parejas en torno a una i-actividad y uso del Foro como herramienta de comunicación asincrónica), un conjunto de episodios de clase que nos permiten abordar los objetivos planteados en este trabajo.

\subsection{Trabajo matemático con la i-actividad}

Los episodios seleccionados para este foco corresponden a parte del trabajo realizado por tres parejas. Su propósito es doble, por un lado, mostrar el rol de la i-actividad como generador de movimientos en los planos verticales de descubrimiento-exploración, y justificación-razonamiento. Mostramos cómo la tecnología ejerce de agente cognitivo que realiza una mediación semiótica en acción entre el plano epistemológico hacía el plano cognitivo de los estudiantes, asumiendo parte del rol de generador de las génesis semiótica e instrumental, y residualmente, la discursiva. Por otro lado, reflejamos cómo las interacciones entre los integrantes de la pareja apuntan a la construcción de un espacio de trabajo común (ETM compartido).

\subsubsection{Episodio 1}

El episodio 1 muestra la influencia de la interactividad con el applet 2 en el proceso de validación de una conjetura en el plano vertical de justificación-razonamiento, promovido por la génesis instrumental. El extracto ejemplifica a su vez cómo la interactividad fomenta la monitorización de las acciones de los estudiantes, gestionando su avance a raíz de dichas acciones de control, ajustando así el ETM idóneo a las restricciones locales.

1- A1: Pues entonces, tenemos un punto ahi, tenemos otro punto aqui,... pues la distancia más corta podríamos decir, a lo mejor que, si como un sistema, pues digamos que puede ser $A X$ y XF. [Al establece una suposición acerca de una posible trayectoria óptima]

2- $\quad$ A2: $\quad$ Ya pero,... ¿Sería recta completamente?, sería si estuviera,

3- $\quad$ A1: $\quad$ Ahí sería [sitúa el punto en el applet 2 con la caja cerrada de tal forma que se visualiza la trayectoria recta (Figura 7)] 


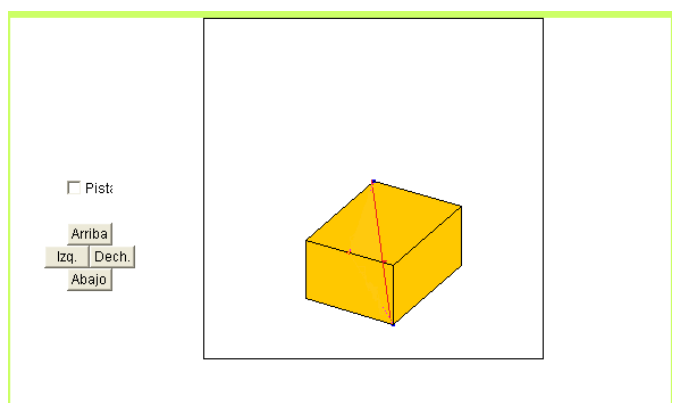

Figura 7 - Applet 2 cerrado y trayectoria recta

4- A2: $\quad$ Si hay tres dimensiones, ... ¿Sería asi?? [Presenta dudas relacionadas con las dimensiones en las que están trabajando]

5- Al: $\quad$ Ahí parece estar recta, ¿no? [no parece caer en la cuenta de la información que parece trasladarle A2]

6- $\quad$ A2: ¿Me entiendes lo que te digo?,..., a ver, sería, hacer..., y luego así. [Señala en la mesa una trayectoria hasta el borde y luego con el dedo desde el borde baja en el espacio "que hay entre la mesa y el suelo". Está reproduciendo lo que observan en la pantalla]. Porque ese cuadro,..., ese cubo tiene tres dimensiones, no dos,... no sé [Espera confirmación de su compañero] ¿Me entiendes lo que te digo?

7- $\quad$ Al: $\quad$ Que si a la hora de poner esto así,... y ampliamos,... y tenemos este cuadrado levantado... [Primero dibuja una trayectoria en tres dimensiones en el papel, a continuación presiona el botón del applet 2 que levanta "el techo" (Figura 8)].
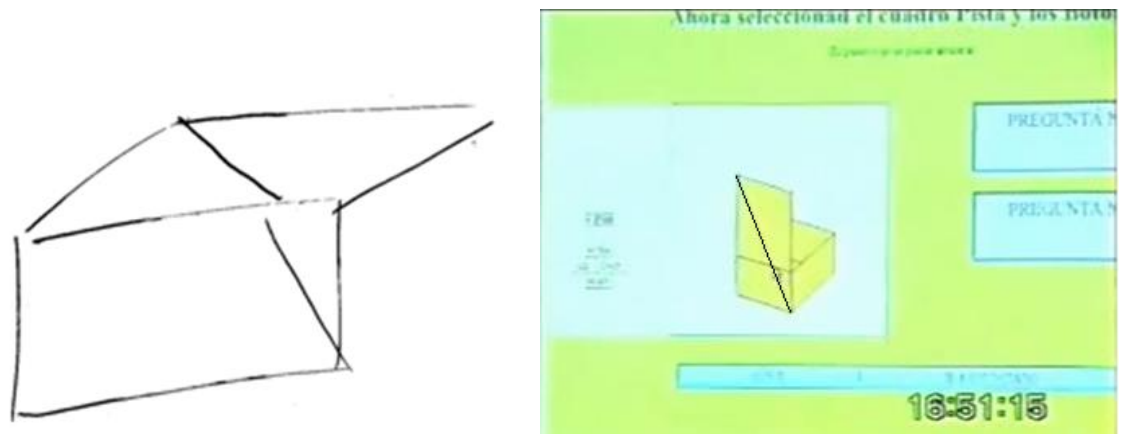

Figura 8 - Dibujo trayectoria y applet 2

8- $\quad$ A2: $\quad$ Aquí [A2 mueve el punto hasta que se visualiza la trayectoria recta]

9- $\quad$ A1: $\quad$ Claro, eso es lo que yo decía, levantarlo para arriba

En los ítems 1, 2 y 5, A1 establece una conjetura, situando la trayectoria sobre el applet 2, de tal forma que parece ser recta, con el techo de la habitación sin levantar. A2 plantea dudas al respecto (ítems 2 y 4). En el ítem 6, A2 intenta explicar al compañero por qué es incorrecto su planteamiento; para eso ejemplifica la trayectoria utilizando la mesa dónde están sentados. Esta validación está motivada por la interacción social y se apoya en la mediación del instrumento. En el ítem 7, A1 dibuja una trayectoria en papel y levanta el techo utilizando el applet, momento en el que se observa que dicha trayectoria no está "recta". En el ítem 8, A2 arrastra la trayectoria hasta que se observa recta y afirma que debe ser ésa. Ambos estudiantes realizan movimientos en el plano vertical descubrimiento-exploración, con 
sucesivas idas y venidas entre el Espacio Local y la Visualización. Como podemos observar, la interacción con el applet gestiona las acciones de los estudiantes, a través de la mediación semiótica en acción (BARRERA, 2013). Este proceso tiene lugar en una dialéctica colaborativa entre los dos estudiantes catalizada por la tecnología digital. En efecto, es lo que se visualiza y el cómo se trasforma lo que hace que los estudiantes lleguen al consenso y admitan la validación de la hipótesis, pasando de este modo al plano vertical justificaciónrazonamiento. En el ítem 9, A1 confirma lo que señala A2, lo que da indicios de la construcción de un ETM compartido.

\subsubsection{Episodio 2}

El episodio 2 vuelve a reflejar el papel de la i-actividad como agente cognitivo y la influencia de la interactividad en el fomento del ETM compartido, a través de la generación de un insight provocado por la interactividad con el applet 2. A diferencia del caso anterior, en esta ocasión hay un movimiento inmediato desde el plano descubrimiento-exploración al plano de justificación-razonamiento.

La pareja, mediante la interacción con el applet 1, había superado el obstáculo asociado a la incapacidad de concebir la existencia de rutas distintas a las que pasan por los vértices del paralelepípedo. A raíz de ello y de responder a la pregunta $n^{\circ}$ 6: “¿Cuál crees que es la trayectoria más corta? ¿Por qué?”, esbozan un plan de resolución en el que detectan la necesidad de calcular las hipotenusas de los triángulos que genera una trayectoria con paso de la hormiga por la arista BC. La secuencia coincide con el instante en el que visualizan por primera vez la página 5 , que contiene el applet 2 .

1- A4: "Activad el cuadro pista",..., Vale. "Presionad los botones y observad". [A4 lee y presiona el botón pista, el paralelepípedo se abre por "el techo" (Figura 9)]

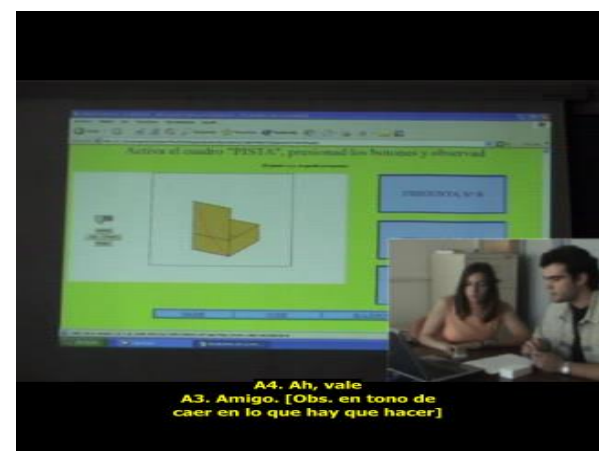

Figura 9 - Fotograma applet 2 
2- $\quad$ A4: $\quad$ iAh!, vale.

3- A3: ¡Amigo! [Ambos estudiantes a la vez, por la mediación semiótica en acción del applet 2, visualizan, en el desarrollo plano parcial del paralelepípedo, cómo dos caras contiguas forman parte de un solo rectángulo y, como consecuencia, se produce un insight]

4- $\quad$ A3: $\quad$ Vale. [Confirma que ya sabe cómo actuar]

5- A4: “Ha cambiado vuestra idea de cuál es el camino más corto?” [A4 inmediatamente descubre el texto de la pregunta 8 y lo lee]

6- $\quad$ A3: $\quad$ Pues va a ser que sí, triángulo rectángulo. Si uno consigue que la hipotenusa sea recta, coges, coges los dos rectángulos, tienes lo mismo y se puede calcular la distancia. [Mientras habla, A3 arrastra el punto rojo sobre la arista y lo sitúa de tal forma que se observa la trayectoria "recta”, a continuación, la pareja calcula el valor de la ruta óptima por la cara frontal del paralelepípedo]

Hasta el inicio del episodio, la i-actividad ha guiado el proceso de resolución de la pareja, fomentando la reflexión a través de las preguntas diseñadas y las oraciones imperativas de acciones sobre el applet 1 , diseñadas para superar el obstáculo asociado a la no consideración rutas distintas de aquellas que pasan por las aristas del paralelepípedo. El Ítem 1 muestra a A4 leyendo la oración imperativa y ejecutando la orden. En ese instante, cuando se abre "el techo", se produce un insight en ambos estudiantes motivado por la interactividad del applet 2 (Ítem 2 y 3). La i-actividad realiza una mediación semiótica en acción que les permite realizar una génesis figural, pasando de la representación a la visualización y de la exploración al descubrimiento. Los ítems siguientes, en los que responden a preguntas de la iactividad, provocan la aparición en el discurso de los estudiantes de un nuevo objeto, el Teorema de Pitágoras, promoviendo una transición directa del plano descubrimientoexploración al de justificación-razonamiento. Así, en el Ítem 4, ambos estudiantes están de acuerdo en el significado de la acción reflejada en el applet. Inmediatamente después de que A3 diga -“Vale”, A4 descubre el texto de la pregunta 8 (Ítem 5). Ello constituye otro indicio de cómo la i-actividad fomenta la construcción de un ETM compartido por los miembros de la pareja y de cómo el ETM idóneo a priori se adapta localmente a este ETM compartido.

\subsubsection{Episodio 3}

El episodio 3 muestra cómo la influencia de la interactividad con el applet 3, la pregunta número $11 \mathrm{y}$ el recuerdo de la estrategia heurística presentada en el applet 2, permiten al estudiante A5 detectar la existencia de rutas alternativas por las caras laterales de la habitación y, a partir de ahí, resolver adecuadamente el problema. De esta manera, la iactividad actúa como catalizador del movimiento entre planos verticales. 
La pareja previamente había calculado la trayectoria óptima por la cara central del paralelepípedo. Justo anterior a este extracto la pareja había interactuado con el applet 3, observando las distintas gráficas de distancia para paso de la hormiga por $\mathrm{X}$ - por la arista central - y X' - por la arista lateral. El diálogo se inicia tras la lectura de la pregunta $\mathrm{n}^{\mathrm{o}} 11$ : “¿Coincide con lo obtenido antes?”.

1- $\quad$ A5: $\quad$ iEs que si tu vuelves a levantar la tapa como antes!, ¿no? [El estudiante A5 se apoya en la producción de gestos metafóricos para otorgar mayor fuerza a sus palabras (Figura 10)]

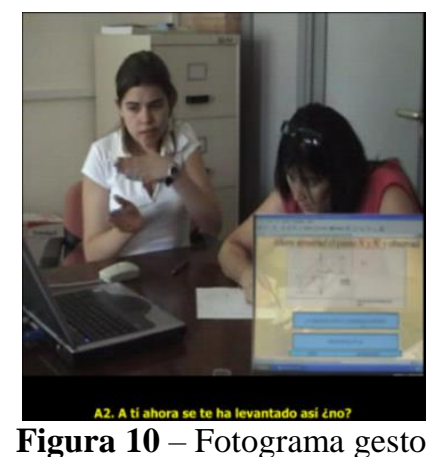

2- A6: $\quad$ ¿Claro!, es que ahora tienes tres y dos, nena,..., mira tres y tres [Esboza en papel la trayectoria por el lateral (Figura 11)]

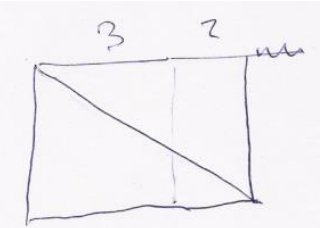

Figura 11 - Dibujo trayectoria lateral

3- A5: ¿Cómo tres y dos?

4- $\quad$ A6: $\quad$ Si yo levanto así para acá, tú tienes tres,..., por aquí, y dos hacia el otro lado. Mira, y entonces tienes que va para acá, tú tienes, tú tienes que va

5- $\quad$ A5: $\quad$ A ti ahora se te ha levantado así ¿no? [Vuelve a realizar el gesto metafórico con las manos]

6- $\quad$ A6: $\quad$ Sí. Y para acá, o sea que puedes, hacer así,..., entonces ahora,..., quizás el camino sea distinto, ¿no? Tres al cuadrado menos,.... [Obtienen la ruta óptima]

En este episodio se refleja cómo la i-actividad permite a la estudiante A5 consolidar la estrategia heurística de "levantar el techo" (desarrollo plano) obtenida de la interacción con el applet 2. En este caso, la influencia de la interactividad es duradera en el tiempo, afianzando su trabajo en el plano de justificación-razonamiento. Esta consolidación es un reflejo de la mediación semiótica de la i-actividad en el ETM personal del estudiante A5, que se traslada al ETM compartido de la pareja, al ser aceptado por su compañera A6.

La imagen del episodio muestra cómo A5 utiliza gestos metafóricos para apoyar su argumentación, situándose de este modo en el plano epistemológico en el que parece hallarse 
su compañera para ayudarle a la transición al plano cognitivo. El Ítem 2 refleja el instante en el que A6 se percata de que la compañera lleva razón (;Claro!). Existe una transferencia de conocimiento entre el ETM personal de A5 hacia el ETM personal de A6 y a un ETM compartido de la pareja (ambos estudiantes "caminan” en la misma dirección). A partir de aquí, A6 se dispone a calcular la distancia de la ruta y la pareja busca el consenso en el modo de proceder, reforzando su ETM compartido (Ítems 3-6).

\subsection{Trabajo matemático mediante e-Learning}

En este apartado se presentan cuatro episodios, seleccionados de entre las interacciones registradas en el Foro de la asignatura, con objeto de mostrar distintas dinámicas producidas entre los ETM idóneo, personales y compartido. Los episodios reflejan a su vez, la modificación de roles por parte de estudiantes y formador y la influencia de las interacciones en estas dinámicas.

\subsubsection{Episodio 4}

El episodio $4^{1}$ muestra a un estudiante que solicita ayuda, al no detectar un error en una actividad relacionada con el uso de la hoja de cálculo para la obtención de una fórmula que relacione datos. Como se puede observar, el estudiante A8 presta ayuda al estudiante A7, mostrando el funcionamiento del operador lógico SI; además añade un consejo general relativo al uso de los paréntesis. La secuencia se desarrolla con A7 en el momento 1 y A8 en el momento 2.

1- $\quad$ A7: $\quad$ hola, tengo un problema con el trabajo 6, en las columnas de condiciones creo que no he hecho algo bien puesto que me sale error, ¿alguien puede decirme en qué me estoy equivocando, por favor?

Vuestras respuestas me serán de gran ayuda, gracias de antemano. Saludos Archivo adjunto: Trabajo_6.ods

2- $\quad$ A8: $\quad H o l a$, en la fórmula del SI() lo que tienes que comprobar es si el contenido de la raíz es menor que 0 , para que si es asíno intente calcularla y te muestre el mensaje que has puesto.

$=\operatorname{SI}\left((\$ C \$ 7 * \operatorname{SENO}(C 12))^{\wedge} 2-4 * 4,9 * 50>=0 ; \operatorname{RAIZ}\left((\$ C \$ 7 * \operatorname{SENO}(C 12))^{\wedge} 2-4 * 4,9 * 50\right)\right.$ ;"NO SE PUEDE")

$Y$ ten cuidado con los paréntesis que si no están bien enlazados tb te dará error. Espero que puedas arreglarlo. Un saludo.

3- $\quad$ A7: $\quad$ Muchas gracias Estudiante A6. Funciona.

\footnotetext{
${ }^{1}$ Los episodios del Foro son una copia fiel de los enviados por los estudiantes, reflejando estrictamente su manera de expresarse y la ortografía utilizada.
} 
La formulación proporcionada del operador SI por parte del estudiante A8 se sitúa en la génesis instrumental puesto que transforma la formulación en el instrumento (en este caso, el lenguaje propio de la Hoja de Cálculo) en el proceso de construcción del significado del operador SI. El episodio 4 muestra una relación direccional entre el ETM del estudiante A8 hacía el ETM del estudiante A7 y hacía un ETM compartido por todos los miembros de la asignatura, a través de la herramienta Foro (Figura 12).

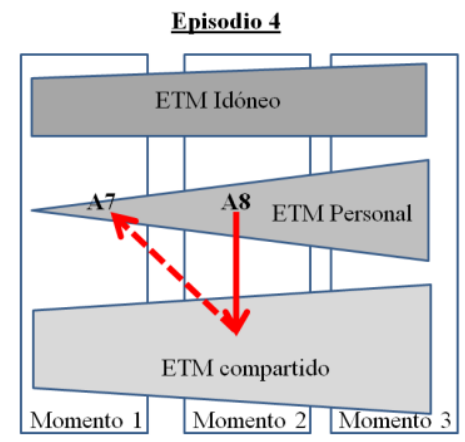

Figura 12 - Movimientos entre los ETM, episodio 4

\subsubsection{Episodio 5}

El episodio 5 refleja cómo el rol del estudiante A10 se modifica, asumiendo el papel de docente al guiar al estudiante A9 durante el proceso de resolución de la actividad, incluso cuando éste parece desear abandonar la tarea (Ítems 5 y 6). En este sentido, es el estudiante A10 quién gestiona y ajusta el ETM idóneo al ETM personal de A9. Por otro lado, hay un aporte conjunto de ambos estudiantes al ETM compartido al presentarse un método de resolución durante la interacción colaborativa. El estudiante A9 está en el momento 1 y A10 en el momento 2. La actividad consiste en estudiar la siguiente sucesión de igualdades en una hoja de cálculo: $1=1 ; 11^{2}=121 ; 111^{2}=12321 ; \ldots$

1- $\quad$ A9: $\quad$ Con respecto al trabajo $n^{\circ} 3$, hay una fórmula que no logro averiguar, que es la que sale en la columna términos y empieza con un numero $1, y$ se te pide una fórmula que al arrastrar la primera celda de la columna donde está el número 1, abajo de ella ponga 11, más abajo 111 y así sucesivamente.

No logro la fórmula que logre esta sucesión de números, si me podrías ayudar os lo agradeceria mucho. Un saludo

2- A10: Fíjate que cada iteración se obtiene con 10,100,1000,... y sumándole la cifra de la iteración anterior. Es decir:

La iteracción 2 es $10+1=11$

La iteracción 3 es $100+11=111$

La iteracción 4 es $1000+111=1111$

Espero haberte servido de ayuda. Un saludo!!

3- $\quad$ A9: Hola! muchas gracias por contestar, pero si haces una fórmula de sumas la cifra anterior más 10, ¿tendrías que aplicar esta fórmula en cada celda no? 
He estado probando con suma con cuadrados y suma de productos pero no me sale nada, lo único la celda B3 que da 11 pero ya no continua la serie

4- $\quad$ A10: Es que tienes que sumarle 10 en la iteracción $2\left(10^{\wedge}(2-1)\right), 100$ en la iteracción $3\left(10^{\wedge}(3-1)\right)$, 1000 en la iteracción $4\left(10^{\wedge}(4-1)\right)$.

Por eso, lo que tienes que sumarle en cada paso depende de la columna izquierda de iteraciones. No se si me explico muy bien.

Un saludo

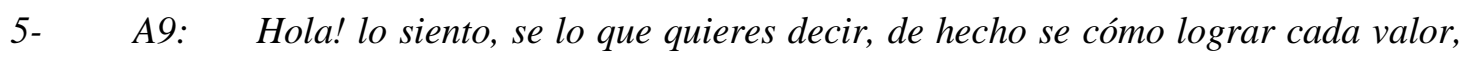
pero el problema lo tengo en la formula. No se cómo expresar la suma, puesto que ¿tendría que 10 elevado a 2 no?, no se si me explico muy bien.

De todas formas, gracias por interesarte

6- A10: Por ejemplo, en la iteración 2, la fórmula sería: $10^{\wedge}(2-1)+1$

Donde el 2 es el número de iteración, es decir, la celda A3 y el 1 que suma es el resultado de la iteración anterior, es decir, la celda $B 2$.

Una vez que tengas eso, al arrastrar hacia abajo Calc va cambiando A3 por $A 4, A 5, A 6 \ldots$ y $B 2$ por $B 3, B 4, B 5 \ldots$

7- $\quad$ A9: $\quad$ Muchisimas gracias, ya lo entendí, de verdad me has ayudado mucho.

El episodio 5 se sitúa en el plano vertical descubrimiento-exploración. Los Ítems 1, 2, 3 y 4 muestran una génesis semiótica desde el representamen de los datos del problema hacía la Visualización de la relación numérica pretendida, mientras que los Ítems 4, 5, 6 y 7 lo hace desde la génesis instrumental y los Artefactos hacía la Construcción, especialmente el Ítem 6 en la que interviene lenguaje específico del tratamiento matemático en hoja de cálculo. Además, este episodio refleja varias relaciones entre ETM. Por un lado, la intervención de A9 apunta a una relación de su ETM personal al ETM compartido y, por el otro, la intervención de A10 guiando a A9 sitúa al primero en el ETM idóneo y refleja una relación hacía el ETM del estudiante A9 y hacía el ETM compartido (Figura 13)

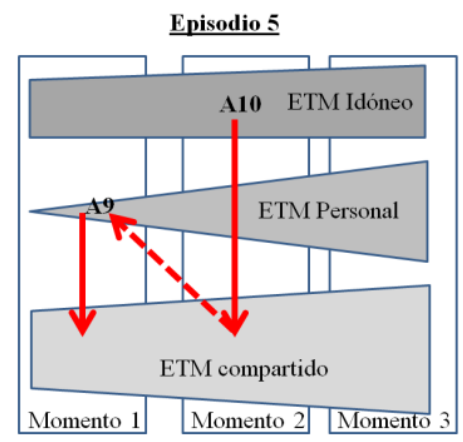

Figura 13 - Movimientos entre los ETM, episodio 5

\subsubsection{Episodio 6}

En relación con esa misma actividad, el episodio 6 presenta una interacción colaborativa entre tres estudiantes (A11, A12 y A13), en la que el estudiante A13 hace referencia a otro mensaje enviado previamente al Foro. En esta ocasión, la situación se 
desarrolla desde el representamen hacía la Visualización, es decir, en la génesis semiótica. Los estudiantes A11 y A12 están en el momento 1, mientras que A13 está en el momento 3.

1- A11: Hola: Alguien me podría ayudar con la fórmula que pasa de 1 a 11 a 111 a $1111 \ldots$ y así sucesivamente. Un saludo y gracias

2- A12: Hola, Yo he multiplicado por 10 y he sumado 1, de la siguiente forma: $1 * 10=10$ y $10+1=11 ; 11 * 10=110$ y $110+1=111 \ldots \ldots$.

Espero servir de ayuda y que encuentres tu propio sistema. Un saludo

3- A13: Hola Estudiante A11, Yo la he hecho como (10^(número de 1's anterior))+(término anterior).

Si quieres echarle un vistazo mejor, mira mi trabajo que lo colgué en un post del foro dedicado a los trabajos $n^{\circ} 3$ y 4 . Saludos.

El episodio 6 refleja varias relaciones entre ETM. La petición de A11 y las respuestas de A12 y A13 apuntan a una relación direccional entre el ETM compartido y el ETM de A11. La intervención de A12 al grupo clase (no responde directamente a A11) apunta a una relación direccional hacía el ETM compartido. Por último, la intervención de A13 apunta a una relación direccional hacía el ETM de A11 y A12 (a éste último también, puesto que el ítem 3 es emitido como una respuesta al ítem 2) y hacía el ETM compartido (Figura 14).

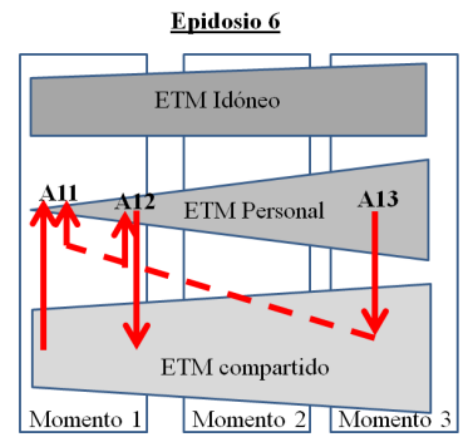

Figura 14 - Movimientos entre los ETM, episodio 6

\subsubsection{Episodio 7}

Por último, el episodio 7 presenta un único mensaje que sitúa al estudiante A14 en la gestión del ETM idóneo, al proponer un método de trabajo alternativo con objeto de visualizar la resolución de la actividad con Geogebra. Ante la dificultad generalizada de los compañeros en el plano de exploración-descubrimiento, propone la utilización de un material físico, anticipándose a una posible intervención del formador. El estudiante A14 está en el momento 3 y la actividad consiste en la obtención de un lugar geométrico (Figura 15). 


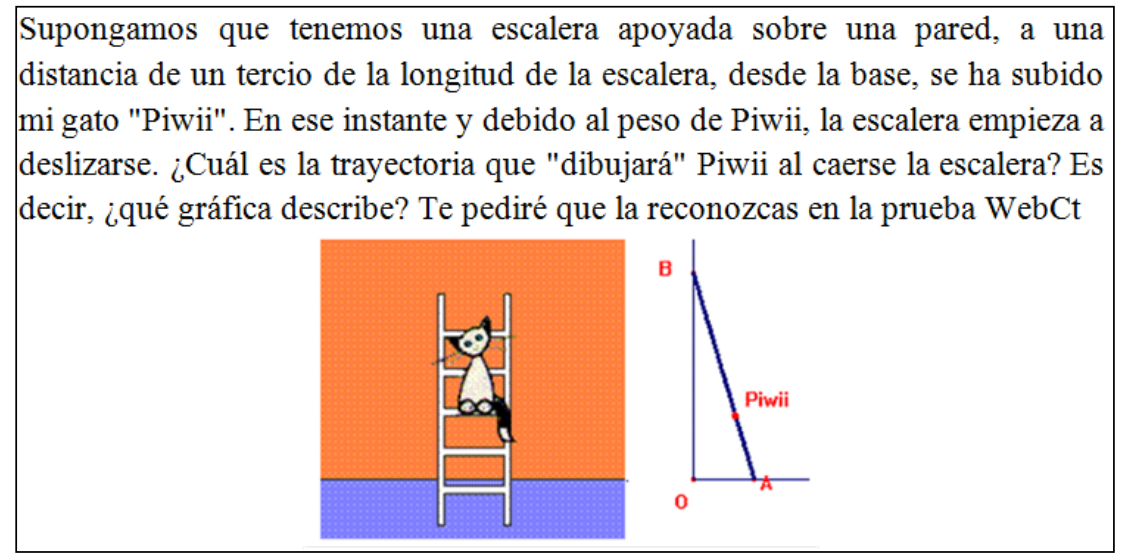

Figura 15 - Enunciado actividad de Piwi

1- $\quad$ A14: $\quad$ Para aquellos que tienen problemas para hacer la actividad de la escalera, os propongo que hagáis una cosa, ya verás como lo veis más claro. Cambia la escalera por un palillo, haz un agujero en él, a un tercio de un lado, de tal forma que quepa en el la punta de un lápiz o boli, y desplázalo por un papel utilizando como pared y suelo los bordes del papel, veras que figura geometrica te sale.

Dicha interacción se sitúa dentro de la génesis figural e indica una relación entre el ETM idóneo, en el que se sitúa el estudiante A14, y el ETM compartido (Figura 16).

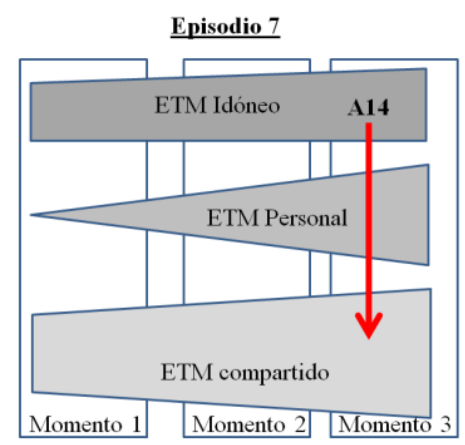

Figura 16 - Movimientos entre los ETM, episodio 7

\section{Conclusiones}

En este artículo mostramos la operatividad del marco de los ETM para dar cuenta del trabajo matemático que se realiza al resolver problemas en un contexto fuertemente mediado por la tecnología. Dicho marco permite describir localmente diferentes dinámicas que tienen lugar entre los planos epistemológico y cognitivo, así como algunos de los efectos que la tecnología tiene en dichas dinámicas. Sin embargo, en un ambiente de aula, esta mirada local ha de ampliarse para abarcar la complejidad de las relaciones entre los distintos ETM de los integrantes del proceso de enseñanza-aprendizaje, las cuales tienen aún potencial por explorar. En nuestro caso, hemos realizado esta exploración en un entorno de e-Learning, en una asignatura del grado de Maestro. 
Los resultados obtenidos muestran, en primer lugar, modificaciones con respecto al papel que se atribuye al docente en la enseñanza presencial dentro del marco de los ETM. Según Gómez-Chacón y Kuzniak (2015) y Barrera (2013), el docente orienta a los estudiantes, especialmente cuando se producen bloqueos y juega un papel fundamental en la guía del estudiante a través de la diversidad de los ETM resultantes de una propuesta didáctica particular. En nuestro caso, el diseño de una i-actividad hace posible que ésta asuma parte de ese papel, ya que asiste a los estudiantes en las diferentes génesis y en las interacciones entre éstas, catalizando el tránsito por distintas etapas del trabajo matemático durante la resolución de un problema; en particular, las asociadas a los planos verticales de descubrimientoexploración y razonamiento-justificación. Ello es posible, no solo por su diseño a priori de un ETM idóneo, sino por su capacidad de ajustarse a los distintos ETM personales y compartidos por las parejas de estudiantes. Así, además de proporcionar heurísticos para la resolución del problema y applets dirigidos a la superación de obstáculos generalizados (applets 1 y 3), es susceptible de adaptarse a los caminos particulares de solución de las parejas de estudiantes (episodios 1 y 2). Ello corresponde a la asunción de subdominios del conocimiento didáctico del contenido del profesor de matemáticas (ROJAS; FLORES; CARRILLO, 2015); en concreto, al conocimiento de la enseñanza de las matemáticas (resolución de problemas) y al conocimiento del aprendizaje de los alumnos (obstáculos y dificultades en contenidos particulares).

Por otra parte, el hecho de trabajar por parejas en torno a la i-actividad fomenta la creación de un ETM compartido en los miembros de la pareja. Indicios de ello son los momentos de convergencia de ETM personales de cada miembro de la pareja en una línea de trabajo común a la que ambos miembros aportan contribuciones (episodio 3). El trabajo compartido se ve potenciado, de un lado, por el hecho de utilizar la misma herramienta y el mismo soporte físico, que centra el trabajo matemático de ambos miembros. De otro lado, la facultad de la i-actividad para guiar el trabajo, proporcionar pistas y realimentar de forma continua, sin representar una autoridad que coarte el libre flujo de expresión del pensamiento de los estudiantes, propicia la producción de interacciones colaborativas entre los miembros de la pareja que engrosan el tejido del trabajo compartido con respecto a entornos no mediados por la tecnología.

La existencia de un ETM compartido se hace quizá más evidente en el caso de la interacción entre los miembros de la clase a través del Foro. Este ETM compartido es socialmente construido a través de las interacciones entre los ETM personales y se registra en 
las herramientas de comunicación del entorno virtual utilizado. El registro contiene, a modo de repositorio, un conglomerado de parcelas de ETM personales que constituyen un ETM común en construcción. Este ETM integra elementos de los planos epistemológico y cognitivo de los ETM personales, junto con procesos propios de las distintas génesis. En este trabajo, hemos ejemplificado algunos de los procesos que tienen lugar en los distintos momentos de ejecución en los que el formador estructura el trabajo matemático (Figura 8). La Figura 8 también ilustra cómo evolucionan los distintos ETM a lo largo de los momentos de ejecución. Así, del momento 1 al momento 3, todos los ETM se enriquecen, incluso el ETM idóneo, que se ve incrementado debido a que, como hemos reflejado, no es una parcela exclusiva del formador, sino que participan en él distintos estudiantes cuando ejercen de guía de sus compañeros/as en las distintas génesis y tránsitos entre ellas.

Para finalizar, cabe plantearse si, en este tipo de entornos, la tecnología puede llegar a suplir, al menos en parte, el papel del docente en la construcción de un ETM idóneo. En nuestra opinión, el papel del docente no se ve mermado, sino que debe transformarse para aprovechar las oportunidades de autonomía y protagonismo que la tecnología brinda a los estudiantes. La cuestión entonces sería cómo los docentes pueden apoyarse de forma efectiva en las tecnologías para crear y gestionar ambientes de trabajo colaborativo, en los que todos los integrantes asuman el protagonismo en la construcción de sus ETM personales y en la contribución a la construcción del ETM idóneo en distintos momentos y de un ETM compartido.

\section{Referencias}

ANGUERA, M. T. Posibilidades y relevancia de la observación sistemática por el profesional de la psicología. Papeles del Psicólogo, Madrid, v. 31, n. 1, p. 122-130, ene. 2010.

BARRERA, R. On the meanings of multiplication for different sets of numbers in context of geometrization: Descartes' multiplication, mathematical workspace and semiotic mediation.

Mathematics Teaching-Research Journal Online, New York, v. 6, n. 1-2, p. 1-20, spring. 2013.

CODINA, A. Teleformación en Educación Matemática. Una experiencia a través de la evaluación formativa y el trabajo colaborativo. In: AGUADED, J. I.; INFANTE, I. (Ed.). Buenas Prácticas en Teleformación en las Diez Universidades Andaluzas. La Coruña: NetBiblo. 2009. p. 303-318.

CODINA, A.; CAÑADAS, M. C.; CASTRO, E. Mathematical problem solving through sequential process analysis. Electronic Journal of Research in Educational Psychology, Almería, v. 13, n. 1, p. 73-110, abr. 2015.

COUTAT, S.; RICHARD, P.R. Les figures dynamiques dans un espace de travail mathématique pour l'apprentissage des propriétés géométriques. Annales de didactique et de sciences cognitives, Strasbourg, v. 16, p. 97-126, 2011. 
DILLENBOURG, P. What do you mean by collaborative learning? In: P. DILLENBOURG (Ed.). Collaborative Learning: cognitive and computational approaches. Oxford: Elservier, 1999, p. 1-19.

ESTRELLA, S.; KUZNIAK, A.; MONTOYA, E.; VIVIER, L. El trabajo matemático en el análisis: una aproximación a los ETM en Francia y Chile. In: GÓMEZ-CHACÓN. I. M. et. al (Ed.).

Mathematical Working Space, Proceedings Fourth ETM Symposium. Madrid: Publicaciones del Instituto de Matemática Interdisciplinar, Universidad Complutense de Madrid, 2015. p. 191-194.

GADANIDIS, G.; GEIGER, V. A social perspective on technology-enhanced mathematical learning: from collaboration to performance. ZDM The International Journal on Mathematics Education, cidade, v. 42, n. 1, p. 91-104, feb. 2010.

GARCÍA, M. M.; ROMERO, I.; GÓMEZ-CHACÓN, I. Procesos de argumentación de estudiantes de secundaria: influencias cognitivas y actitudinales. In: GÓMEZ-CHACÓN, I. M. et. al (Ed..)

Mathematical Working Space, Proceedings Fourth ETM Symposium. Madrid: Publicaciones del Instituto de Matemática Interdisciplinar, Universidad Complutense de Madrid, 2015. p. 421-444.

GÓMEZ-CHACÓN, I.; KUZNIAK, A. Spaces for geometric work: figural, instrumental, and discursive geneses of reasoning in a technological environment. International Journal of Science and Mathematics Education, Taipei, v. 13, n. 1, p. 1-26, feb. 2015.

HOUDEMENT, C.; KUZNIAK, A. Paradigmes géométriques et enseignement de la géométrie. Annales de Didactique et de Sciences Cognitives, Strasbourg, v. 11, p. 175-193, 2006.

JOCHEMS, W. M. G; MARTENS, R. L.; STRIJBOS, J. W. Designing for interaction: six steps to designing computer-supported group-based learning. Computers \& Education, Taipei, v. 42, n. 4, p. 403-424, may. 2004.

KUZNIAK, A. Espace de travail géométrique personnel: une approche didactique et statistique. In: III INTERNATIONAL CONFERENCE IMLICATIVE STATISTIC ANALYSIS, $3^{\text {th }}, 2005$. Palermo. Proceedings... Palermo,: Università degli studi di Palermo, 2005, p. 211-227.

KUZNIAK, A. L'espace de travail mathématique et ses genèses. Annales de Didactique et de Sciences Cognitives, Strasbourg Cedex, n. 16, 2011, p. 9-24, 2011.

KUZNIAK, A.; RICHARD, P. R. Espacios de trabajo matemático. Punto de vista y perspectivas. Relime, México D. F., v. 17, n.4-I, p. 5-15, 2014.

MENA, A.; MORALES, A. Elementos para una aproximación epistemológica a un "espacio de trabajo" algebraico. In: XIII CONFERENCIA INTERAMERICANA DE EDUCACIÓN MATEMÁTICA, 12 $2^{\text {th }}, 2011$, Recife. Actas... Recife: Universidade Federal de Pernambuco, 2011, p. $1-12$.

MISKULIN, R. G. et al.. A prática do professor que ensina matemática e a colaboração: uma reflexão a partir de procesos formativos virtuais. Bolema, Rio Claro (SP), v. 25, n. 41, p. 173-186, dez. 2011.

MITHALAL, J. Initier un processus de preuve mathématique dans un environnement de géométrie dynamique 3D. Relime, México D. F., v. 17, n.4-II, p. 343-360, 2014.

MONTOYA, E. Los paradigmas geométricos en la formación inicial de profesores de Matemática. In: XIII CONFERENCIA INTERAMERICANA DE EDUCACIÓN MATEMÁTICA, 13 ${ }^{\text {th }}$, 2011,Recife.

Actas... Recife: Universidade Federal de Pernambuco, 2011. p. 1-13. 
BARRA, M; et al.. El espacio de trabajo matemático: una herramienta de análisis. XXXVIII SEMANA DE LA MATEMÁTICA PUCV, edição, ano, local. Anais... Valparaiso: Pontificia Universidad Católica de Valparaiso, 2012. p. 1-24

MOSCHOKOVICH, J. N.; BRENNER, M. E. Integrating a naturalistic paradigm into research on mathematics and science cognition and learning. In: KELLY, A. E.; LESH, R. (Ed.). Handbook of Research Designs in Mathematics and Science Education. Mahwah: Lawrence Erlbaum Associates, 2000. p. 457-486.

ROJANO, T.; MORENO, L. Educación matemática: investigación y tecnología en el nuevo siglo. Avance y Perspectiva, México D.F., , n. 18, p.325-334, sept. 1999.

ROJAS, N.; FLORES, P.; CARRILLO, J. Conocimiento especializado de un profesor de matemáticas de educación primaria al enseñar los números racionales. Bolema, Rio Claro (SP), v. 29, n. 51, p.143166, abr. 2015.

SEDIG, K.; SUMMER, M. Characterizing interaction with visual mathematical representations. International Journal of Computers for Mathematical Learning, New York, v. 11, n. 1, p.1-55, apr. 2006.

SIMS, R. Interactivity: A forgotten art? Instructional Technology Research Online Repository, 1997, Disponible en: $<$ http://www2.gsu.edu/ wwwitr/ $>$. Accesado en: 7 mar. 2015.

TESSIER-BAILLARGEON, M. et al.. Conception et analyse de geogebratutor, un système tutoriel intelligent: genèse d'un espace de travail géométrique idoine. Relime, México D. F., v. 17, n.4-II, p. 303-326, 2014.

WALDEGG, G. El uso de las nuevas tecnologías para la enseñanza y el aprendizaje de las ciencias. Revista electrónica de Investigación Educativa, Tijuana, México, v. 4, n. 1, p. 1-22, 2002.

Disponible en: <http://redie.uabc.mx/index.php/redie/article/view/53/97>. Accesado en: 15 may. 2005.

WERTSCH, J. Voices of the mind. A sociocultural approach of mediated action. London: Harveser, 1991.

ZAMBRANO, W. R.; MEDINA, V.H.; GARCIA, V. M. Nuevo rol del profesor y del estudiante en la Educación Virtual. Dialéctica Revista de Educación, Bogotá, n. 26, p. 51-61, 2010.

Submetido em Julho de 2015. Aprovado em Setembro de 2015. 